Research Paper

\title{
The Prognostic Value of Tyrosine Kinase SRC Expression in Locally Advanced Rectal Cancer
}

\author{
Felix Rühlmann1, Manuel Nietert², Thilo Sprenger ${ }^{1}$, Hendrik A. Wolff3, ${ }^{3}$, Kia Homayounfar1, Peter Middel 5 , \\ Hanibal Bohnenberger ${ }^{6}$, Tim Beissbarth ${ }^{2}$, B. Michael Ghadimi ${ }^{1}$, Torsten Liersch ${ }^{1}$, Lena-Christin Conradi ${ }^{1 凶}$ \\ 1. Department of General, Visceral, and Pediatric Surgery, University Medical Center, Göttingen, Germany; \\ 2. Department of Medical Statistics, University Medical Center, Göttingen, Germany; \\ 3. University Medical Center, Göttingen, Germany; \\ 4. Radiologie München, München, Germany; \\ 5. Pathology Nordhessen, Kassel, Germany; \\ 6. Department of Pathology, University Medical Center, Göttingen, Germany. \\ $\triangle$ Corresponding author: Lena-Christin Conradi, M.D.,General, Visceral and Pediatric Surgery, University Medical Center Göttingen, Robert-Koch-Str. 40, \\ D-37075, Göttingen, Germany tel: +49-551-39-10480; fax: +49-551-39-9450 e-mail: lena.conradi@med.uni-goettingen.de \\ (c) Ivyspring International Publisher. This is an open access article distributed under the terms of the Creative Commons Attribution (CC BY-NC) license \\ (https://creativecommons.org/licenses/by-nc/4.0/). See http://ivyspring.com/terms for full terms and conditions.
}

Received: 2016.07.25; Accepted: 2017.01.09; Published: 2017.04.10

\begin{abstract}
The cellular sarcoma gene (SRC) is a proto-oncogene encoding for a tyrosine kinase. SRC expression was determined in locally advanced rectal adenocarcinoma tissue from pretreatment biopsies and resection specimens. The expression level was correlated with clinicopathological parameters to evaluate the predictive and prognostic capacity.

For this monocentric analysis 186 patients with locally advanced rectal cancer (median: 63.7 years; 130 men (69.9\%), 56 women (30.1\%)) were included. Patients with a carcinoma of the upper third of the rectum were treated with primary tumor resection $(n=27 ; 14.5 \%)$. All other patients received a preoperative chemoradiotherapy (CRT) with 50.4 Gy and concomitant 5-fluorouracil (5-FU) or 5-FU+oxaliplatin followed by postoperative chemotherapy with 5-FU or 5-FU+oxaliplatin. SRC expression was determined with immunohistochemical staining from pretreatment biopsies $(n=152)$ and residual tumor tissue from the resection specimens $(n=163)$. The results were correlated with clinicopathological parameters and long-term follow-up.

The expression of SRC was determined in pretherapeutic biopsies (mean H-Score: 229) and resection specimens (mean H-Score: 254). High SRC expression in pretherapeutic tumor samples significantly correlated with a negative postoperative nodal status $(p=0.005)$. Furthermore an increased protein expression in residual tumor tissue was associated with fewer distant metastases $(p=0.04)$. The overexpression of SRC in pretreatment tumor biopsies showed also a trend for a longer cancer-specific survival (CSS; $p=0.05)$ and fewer local relapses $(p=0.06)$ during long-term follow-up.

High SRC expression in rectal cancer seems to be associated with a better long-term outcome. This finding could help in the future to stratify patients for a recurrence risk adapted postoperative treatment.
\end{abstract}

Key words: SRC, rectal cancer, tyrosine kinase, preoperative chemoradiotherapy, biomarker, targeted therapy.

\section{Introduction}

Colorectal Cancer (CRC) is one of the most frequent cancers in the western world. It represents the third most common cause of cancer-related deaths in the USA and in Europe it was estimated that more than 440,000 people were diagnosed with CRC in 2012.1,2 Within the last two decades, the standard treatment of locally advanced Union International contre le Cancer (UICC) II/III rectal cancer consists of 
a preoperative 5-FU-based chemoradiotherapy (CRT) followed by total mesorectal excision (TME) ${ }^{3-5}$ because it was significantly associated with improved locoregional control, rate of sphincter preservation and long-term survival with favourable acute and late toxicity profiles compared to postoperative CRT.6-9

Although advances in chemotherapy and the development of specific antibody therapies, surgery as well as radiation therapy have been made, long-term outcome and disease-free survival rates still have not improved.10 The long-term prognosis is limited due to the fact that $30 \%$ to $40 \%$ of patients with CRC still develop distant metastases in the further course of disease.3,11,12 Furthermore, each treatment modality has its own side effects, which can be severe and an even intensified therapy can compromise patients' quality of life. Thus, an individualised risk-adapted treatment for each individual patient would be desirable. However, until today, no reliable biomarker has been established in consideration of response prediction to preoperative CRT and individual prognosis in the clinical routine. ${ }^{13-16}$

Clinical trials (e.g. CAO/ARO/AIO-04 trial; study number: NCT00349076) have been testing intensified preoperative CRT protocols in order to improve local treatment efficacy and postoperative chemotherapy (CTx). The aim is to prolong disease-free survival (DFS) to ultimately enable a better long-term outcome. ${ }^{17-22}$ However until today, we cannot clearly predict which patient benefits from an intensified therapy. This is why the implementation of biomarkers in clinical settings is needed.

The SRC gene is a proto-oncogene encoding for the SRC tyrosine kinase. ${ }^{23}$ Though its role in cancer is still not fully understood, an overexpression of the SRC protein as well as an increase in its specific activity has been observed in numerous cancer types. ${ }^{24,25}$ Especially gastrointestinal cancer types like colorectal cancer show increased SRC activity. ${ }^{26}$ SRC plays a central role in the regulation of cell differentiation, adhesion, migration, invasion, proliferation, angiogenesis and immune function. ${ }^{24,27-28}$

Therefore the role of SRC expression considering its predictive and prognostic potential in locally advanced rectal cancer tissue was analysed.

For this, the expression of SRC was assessed in pretherapeutic biopsies and residual tumour tissue of locally advanced stage (cUICC II/III) rectal cancer after CRT and TME surgery in 186 patients using immunohistochemical staining (see figure 1).

\section{Materials and Methods}

\section{Patient cohort}

One hundred and eighty-six consecutive patients (median: 63.7 years; 130 men $(69.9 \%)$, 56 women $(30.1 \%)$ ) with rectal adenocarcinomas clinically diagnosed as UICC II and III were included in this monocentric retrospective analysis. All patients were treated within phase-II/III trials of the German Rectal Cancer Study Group (e.g. CAO/ARO/AIO-94, CAO/ARO/AIO04). 3,29-30 Among these patients, 103 (55.4\%) were treated with preoperative CRT with 5 -FU or with 5-FU + oxaliplatin ( $\mathrm{n}=56 ; 30.1 \%)$. Another 27 patients (14.5\%) received primary surgery followed by postoperative CRT or in case of a tumor localization in the upper third of the rectum $(12$ to $16 \mathrm{~cm})$ by postoperative CTx with 5-FU + oxaliplatin (GAST-05 trial). The eligibility criteria included histologically confirmed adenocarcinoma and localization within $16 \mathrm{~cm}$ above the anocutaneous verge measured by rigid rectoscopy. ${ }^{31}$ Staging
Figure 1. Study design. This figure shows the treatment of patients and time points of tissue collection for analysis of SRC expression. CEA, carcinoembryonic antigen; CT, contrast-enhanced computer tomography; EUS indicates endorectal ultrasound; MRI, magnetic resonance imaging; CSS, Cancer specific survival; DFS, Disease free survival; ox, oxaliplatin. 
procedures included endorectal ultrasonography, contrast-enhanced computer tomography (CT) scans of the abdomen and pelvis and/or magnetic resonance imaging (MRI) of the pelvis in order to confirm locally advanced but resectable rectal cancer. Every patient was treated at the Department of General, Visceral and Pediatric Surgery, University Medical Center Göttingen (UMG), Germany, between May 1998 and February 2010. Clinicopathological parameters of all patients and histopathological characteristics are summarized in table 1 . The informed consent for additional translational research projects and the approval from the ethics committee of the UMG were obtained from all patients before enrollment into this study (ethics committee approval number $9 / 8 / 08$ ).

\section{CRT and Surgery - Multimodal Treatment}

Irradiation for patients receiving preoperative CRT was delivered with a total dose of $50.4 \mathrm{~Gy}^{32}$ in 28 fractions of $1.8 \mathrm{~Gy}$ as 3-dimensional conformal irradiation, by intensity-modulated radiotherapy or volumetric intensity-modulated arc therapy with 6 or 20 megavolt (MV) photons. ${ }^{33}$ In 103 patients receiving 5-FU based CRT, 5-FU was applied as 120-hour infusion of $1000 \mathrm{mg} / \mathrm{m}^{2} / \mathrm{d}$ during weeks 1 and 5 . The same regimen was performed postoperatively to the patients getting postoperative CRT. In 56 patients being randomized to an intensified chemotherapy regimen, preoperative CRT was applied as combination of 5 -FU $\left(250 \mathrm{mg} / \mathrm{m}^{2} / \mathrm{d}\right)$ on days 1 to 14 and 22 to 35 and oxaliplatin $\left(50 \mathrm{mg} / \mathrm{m}^{2} / \mathrm{d}\right)$ on days 1 , 8,22 and 29. Six weeks after completion of preoperative CRT, quality-controlled TME surgery was performed.

After four to six weeks after surgery, multimodal treatment was completed with 4 cycles of 5-FU $\left(500 \mathrm{mg} / \mathrm{m}^{2} / \mathrm{d}\right.$ ) bolus infusions (days 1 to 5 ) or with 4 cycles of 5 -FU $\left(2400 \mathrm{mg} / \mathrm{m}^{2}\right)$ continuous infusion combined with oxaliplatin $\left(100 \mathrm{mg} / \mathrm{m}^{2}\right)$ and folinic acid $\left(400 \mathrm{mg} / \mathrm{m}^{2}\right)$ infusion.

\section{Tumor Regression Grading and Histopathologic Staging}

The semiquantitative 5-point Dworak tumor regression grading (TRG) system, classifying residual tumor tissue, was used as an early surrogate parameter in order to estimate therapeutic response to preoperative CRT. ${ }^{6,34}$ Histopathologically confirmed pathological Complete Response ( $\mathrm{pCR}$ ) was defined as absence of any vital tumor cells in the area of the primary cancer and of all detected lymph nodes (ypTON0).
Table 1. Clinicopathologic parameters and Histopathologic Characteristics of Examined Patients $(n=186)$

\begin{tabular}{|c|c|c|c|c|}
\hline $\begin{array}{l}\text { Parameters/ } \\
\text { Characteristics }\end{array}$ & $\begin{array}{l}\text { Primary } \\
\text { surgery } \\
(n=27)\end{array}$ & $\begin{array}{l}\text { Preoperative } \\
\text { CRT with } \\
\text { 5-FU (n=103) }\end{array}$ & $\begin{array}{l}\text { Preoperative } \\
\text { CRT with 5-FU } \\
+ \text { oxaliplatin } \\
(\mathrm{n}=56)\end{array}$ & $\begin{array}{l}\text { p-value } \\
\dagger\end{array}$ \\
\hline Age [years] & & & & $0.61 \#$ \\
\hline Mean & 64.9 & 63.6 & 62.4 & \\
\hline Range & $44-81$ & $35-83$ & $37-82$ & \\
\hline Sex & & & & 0.56 \\
\hline Male & $17(63 \%)$ & $75(73 \%)$ & $38(68 \%)$ & \\
\hline Female & $10(37 \%)$ & $28(27 \%)$ & $18(32 \%)$ & \\
\hline \multicolumn{2}{|c|}{$\begin{array}{l}\text { Tumor localization (above } \\
\text { anocutaneous verge) }\end{array}$} & & & 0.08 \\
\hline $0-6 \mathrm{~cm}$ & $9(33 \%)$ & $45(44 \%)$ & $23(41 \%)$ & \\
\hline$>6-12 \mathrm{~cm}$ & $12(44 \%)$ & $51(50 \%)$ & $31(55 \%)$ & \\
\hline$>12-16 \mathrm{~cm}$ & $6(22 \%)$ & $6(6 \%)$ & $2(4 \%)$ & \\
\hline cT stage & & & & 0.01 \\
\hline $\mathrm{T} 1$ & $1(4 \%)$ & - & - & \\
\hline $\mathrm{T} 2$ & $5(19 \%)$ & $5(5 \%)$ & $1(2 \%)$ & \\
\hline T3 & $19(70 \%)$ & $92(89 \%)$ & $54(96 \%)$ & \\
\hline T4 & $2(7 \%)$ & $6(6 \%)$ & $1(2 \%)$ & \\
\hline cN stage & & & & 0.29 \\
\hline positive $(\mathrm{cN}+)$ & $17(63 \%)$ & $72(70 \%)$ & $41(73 \%)$ & \\
\hline negative $(\mathrm{cN}-)$ & $10(37 \%)$ & $30(29 \%)$ & $15(27 \%)$ & \\
\hline unknown & - & $1(1 \%)$ & - & \\
\hline cUICC stage & & & & 0.02 \\
\hline I & $3(11 \%)$ & - & - & \\
\hline II & $7(26 \%)$ & $31(30 \%)$ & $12(21 \%)$ & \\
\hline III & $15(56 \%)$ & $70(68 \%)$ & $41(73 \%)$ & \\
\hline IV & $2(7 \%)$ & $2(2 \%)$ & $3(5 \%)$ & \\
\hline Surgical procedure & & & & 0.27 \\
\hline $\begin{array}{l}\text { Low anterior } \\
\text { resection }\end{array}$ & $20(71 \%)$ & $61(59 \%)$ & $43(77 \%)$ & \\
\hline $\begin{array}{l}\text { Abdominoperineal } \\
\text { resection }\end{array}$ & $6(21 \%)$ & $38(37 \%)$ & $12(21 \%)$ & \\
\hline $\begin{array}{l}\text { Hartmann } \\
\text { procedure }\end{array}$ & $2(7 \%)$ & $4(4 \%)$ & $1(2 \%)$ & \\
\hline Resection margin & & & & 0.03 \\
\hline R0 & $25(93 \%)$ & $102(99 \%)$ & $56(100 \%)$ & \\
\hline R1 & $2(7 \%)$ & $1(1 \%)$ & - & \\
\hline TRG & & & & 0.68 \\
\hline 0 & - & - & - & \\
\hline 1 & - & $14(14 \%)$ & $5(9 \%)$ & \\
\hline 2 & - & $30(29 \%)$ & $14(25 \%)$ & \\
\hline 3 & - & $51(49 \%)$ & $33(59 \%)$ & \\
\hline 4 & - & $8(8 \%)$ & $4(7 \%)$ & \\
\hline (y)pT stage & & & & 0.47 \\
\hline 0 & $1(4 \%)$ & $8(8 \%)$ & $4(7 \%)$ & \\
\hline 1 & $2(7 \%)$ & $5(5 \%)$ & $9(16 \%)$ & \\
\hline 2 & $9(33 \%)$ & $25(24 \%)$ & $12(21 \%)$ & \\
\hline 3 & $13(48 \%)$ & $59(57 \%)$ & $28(50 \%)$ & \\
\hline 4 & $2(7 \%)$ & $6(6 \%)$ & $3(6 \%)$ & \\
\hline (y)pN stage & & & & 0.1 \\
\hline 0 & $16(59 \%)$ & $64(62 \%)$ & $43(77 \%)$ & \\
\hline 1 & $5(19 \%)$ & $23(22 \%)$ & $11(20 \%)$ & \\
\hline 2 & $6(22 \%)$ & $16(16 \%)$ & $2(3 \%)$ & \\
\hline (y)pUICC stage & & & & 0.48 \\
\hline 0 & $1(4 \%)$ & $7(7 \%)$ & $4(7 \%)$ & \\
\hline I & $8(30 \%)$ & $21(20 \%)$ & $20(36 \%)$ & \\
\hline II & $7(26 \%)$ & $33(32 \%)$ & $16(28 \%)$ & \\
\hline III & $9(33 \%)$ & $35(34 \%)$ & $11(20 \%)$ & \\
\hline IV & $2(7 \%)$ & $7(7 \%)$ & $5(9 \%)$ & \\
\hline Cancer recurrence & & & & 0.83 \\
\hline None & $17(63 \%)$ & $69(67 \%)$ & $39(70 \%)$ & \\
\hline Local & $3(11 \%)$ & $1(1 \%)$ & $1(1 \%)$ & \\
\hline Distant & $7(26 \%)$ & $33(32 \%)$ & $16(29 \%)$ & \\
\hline \multicolumn{5}{|l|}{ Distant metastases } \\
\hline Hepatic & $1(14 \%)$ & $9(27 \%)$ & $6(38 \%)$ & \\
\hline Pulmonary & $4(57 \%)$ & $12(36 \%)$ & $2(12 \%)$ & 0.25 \\
\hline Peritoneal / other & $2(29 \%)$ & $12(36 \%)$ & $8(50 \%)$ & \\
\hline \multicolumn{5}{|l|}{ Follow-up time } \\
\hline Median & 110,0 & 60,0 & 48,5 & \\
\hline Range & $21-163$ & $1-163$ & $3-110$ & \\
\hline Mean & 96,3 & 66,4 & 49,0 & \\
\hline Standard deviation & 40,7 & 37,1 & 24,1 & \\
\hline $\begin{array}{l}\text { Cancer related } \\
\text { death }\end{array}$ & $7(26)$ & $10(18)$ & $19(18)$ & \\
\hline
\end{tabular}


Histopathological staging included current TNM stage classification. 35,36 Nodal staging was evaluated histopathologically examining all detected lymph nodes and the determination of the lymph node ratio in all cases.

\section{Study Cohorts for SRC Analysis}

Tumor tissue was collected at two different time points: first during diagnostic biopsy procedure and second at the time of TME surgery. Pretreatment biopsies fulfilling the minimal quality criteria $(\geq 50$ carcinoma cells) were available from 152 patients. Resection specimens with $\geq 100$ carcinoma cells were suitable for evaluation and were available from 163 samples. As a result 129 matched pairs of biopsies and corresponding resection specimens were identified.

\section{Determination of SRC Status}

The SRC status was assessed using paraffin-embedded tissue samples, which were cut into slices of $2 \mu \mathrm{m}$. Using the monoclonal rabbit antibody anti-SRC (36D10) Rabbit $m A b$ (Cell Signal, Boston, MA, 1:2000 diluted), a standardized immunohistochemical staining technique was performed on a Ventana Bench-Mark XT immunostainer (Ventana, Tucson, AZ). The heat epitope retrieval was performed for 60 minutes at $100^{\circ} \mathrm{C}(\mathrm{pH}=9)$. The anti-SRC antibody was incubated at $37^{\circ} \mathrm{C}$ for 30 minutes. The enzymatic reactivity was visualized by means of horseradish peroxidase using the ultraView Universal DAB Detection Kit (Ventana Medical Systems).

As SRC is localized in the cytosol as well as in the membrane, both staining localizations were analyzed. For cytoplasmic staining, four different staining intensity grades were defined: 0 (no staining), 1 (weak staining), 2 (strong staining), 3 (very strong staining). For membrane staining, another four grades were defined: 3 (differentiation of membrane staining at low magnification (5x) was possible), 2 (differentiation of membrane staining at medium magnification (20x) was possible), 1 (differentiation of membrane staining at high magnification (40x) was possible), 0 (even at highest magnification (40x) no differentiation of membrane staining was possible).

\section{Follow-up}

Follow-up examinations of all patients were performed at 3-month intervals within the first 2 years and at 6-month intervals after 2 years for a period of 10 years $^{31}$, according to the study protocols or national guidelines. ${ }^{30,31}$ DFS was defined as the period between surgical resection of the primary and any evidence of cancer recurrence. CSS was defined as the interval between the surgical resection of the primary rectal cancer and cancer-related death.

\section{Statistical Analysis}

SRC expression was correlated with clinicopathological parameters (uT, uN, TRG, (y)pT, (y)pN, M status, (y)pUICC) and DFS, CSS and overall survival (OS). The workflow for the preprocessing and the analysis of the data was implemented using KNIME 2.7.0.37 KNIME nodes were mostly used for the preprocessing of the data, while we used the R-plugin nodes in KNIME to perform the final statistical tests with $\mathrm{R}$ version 3.0.2. The global significance level was set to $a=5 \%$. For comparisons of continuous data we used the Pearson's correlation coefficient ( $r$ ). If the data was skewed we used the non-parametric, rank based correlation coefficient (tau) according to Kendall. For comparisons of two continuous data distributions we used the Wilcoxon rank sum test, paired where applicable. In case of three or more different distribution samples we used the Kruskal-Wallis rank sum test for the comparison. The impact of SRC on DFS, CSS was determined using Kaplan-Meier analysis and assessed for statistical significance using the log rank test (as Cox proportional hazard model with binary input) and where applicable for the continuous data values using a Cox proportional hazard model. The survival analysis was performed using the R package survival.

In order to quantify immunohistochemical staining the H-Score was implemented, ranging from 0 (weakest staining) until 300 (strongest staining) by multiplicating intensity of staining with the percentage of stained tissue. ${ }^{38}$

\section{Results}

\section{Patient Characteristics and Recurrence}

With respect to the primary tumor localization of rectal adenocarcinoma at the time of pretherapeutic staging (table 1), $78(41.9 \%)$ tumors were localized in the lower third $(0-6 \mathrm{~cm}), 94(50.5 \%)$ tumors were localized in the middle third $(>6-12 \mathrm{~cm})$, and $14(7.5 \%)$ tumors were localized in the upper third $(>12-16 \mathrm{~cm})$ of the rectum. The surgical procedures performed were $126(67.7 \%)$ low anterior resections, 56 (30.1\%) abdominoperineal resections and 4 discontinuous resections (Hartmann's procedure) (2.2\%), all including TME. Considering the efficacy of preoperative CRT in this study, the pretherapeutic cUICC staging and postoperative ypUICC tumor staging results are summarized in table 1 . The median follow-up was 71.6 months. The local and/or distant cancer recurrences were observed in 51 (32.0\%) patients after preoperative CRT and in $10(37.0 \%)$ patients after primary surgery followed by postoperative $C(R) T$ (table 1). In 56 patients having distant metastases, the predominant localizations of 
cancer relapse were pulmonal in $18(32.1 \%)$ cases and hepatic metastases in 16 patients $(28.6 \%)$

\section{Pretherapeutic SRC expression and prognosis}

SRC expression was overall higher in the resection specimens compared to the expression in pretherapeutic biopsies (mean H-Score in biopsies: 229; mean H-score in resection specimen: 254). A high pretherapeutic SRC expression level was associated with a negative postoperative nodal status $(p=0.005$; see figure 3A). Furthermore, there was a non-significant trend for prolonged CSS $(p=0.059)$ and fewer local relapses $(p=0.06)$ during long-term follow-up. In addition, even in patients with a
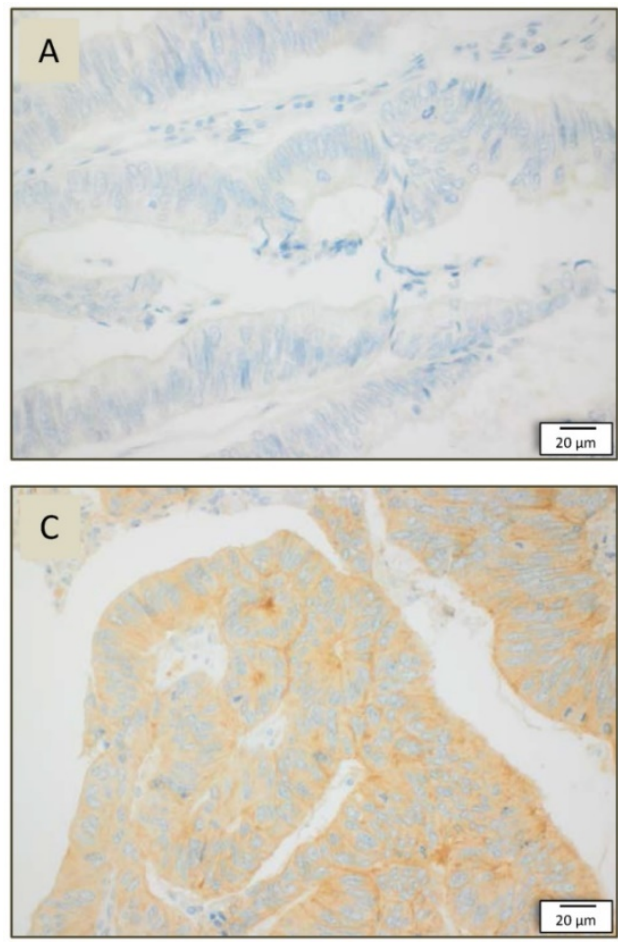

\begin{tabular}{|l|lrll|}
\hline & Gender & Age & Histopathologylogy (TNM) & Resection \\
\hline SRC 0 & male & 62 & pT2, pNO (0/24), cM0, G2, R0 & Low anterior resection \\
\hline SRC I & male & 46 & ypT3b, pNO (0/54), cM0, G2-3, R0 & Low anterior resection \\
& & & & \\
\hline SRC II & female & 67 & pT2, pNO (0/17), cM0, G2, R0 & Low anterior resection \\
& & & & \\
\hline SRC III & male & 57 & ypT2, ypNO (0/27), cM0, G2, R0 & Low anterior resection \\
\hline
\end{tabular}

Figure 2. Images of immunhistochemical staining in resection specimens of rectal cancer: A SRC cytosolic staining intensity 0 in $40 x$ magnification, B SRC cytosolic staining intensity in 40x magnification, C SRC cytosolic staining intensity in 40x magnification, D SRC cytosolic staining intensity III in 40x magnificationon.
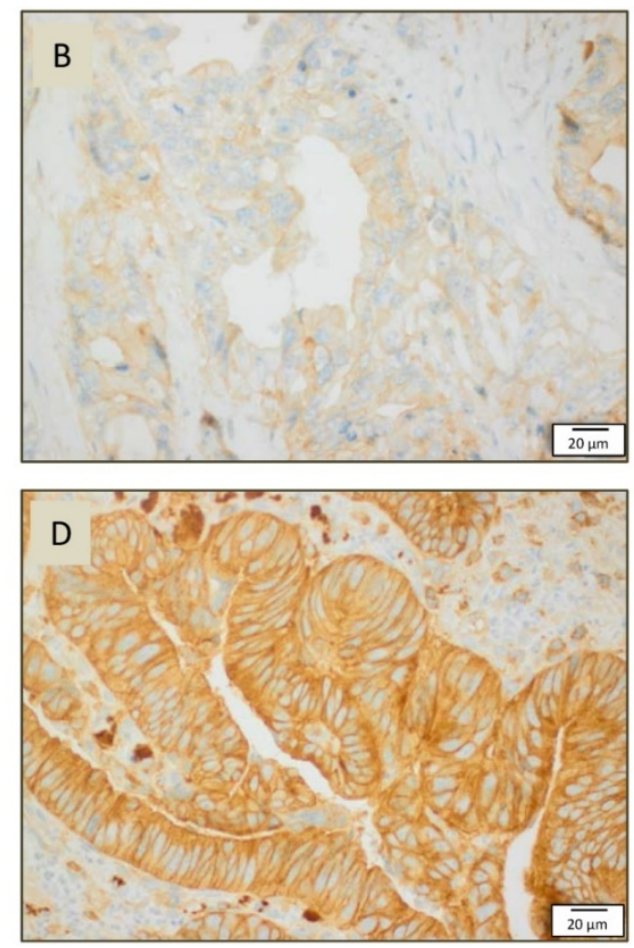

positive nodal status, increased SRC expression was associated with a longer DFS ( $\mathrm{p}=0.026)$. Besides, these patients also developed fewer distant metastases $(\mathrm{p}=0.04)$. Patients who underwent preoperative CRT with 5-FU + oxaliplatin with a high SRC expression in pretherapeutic biopsies showed more often a negative nodal status compared to those patients who were treated with RT and 5-FU alone (see figure 3B). The current study could not reveal a significant association between the pretherapeutic SRC expression level and the CRT-induced histopathological TRG. 


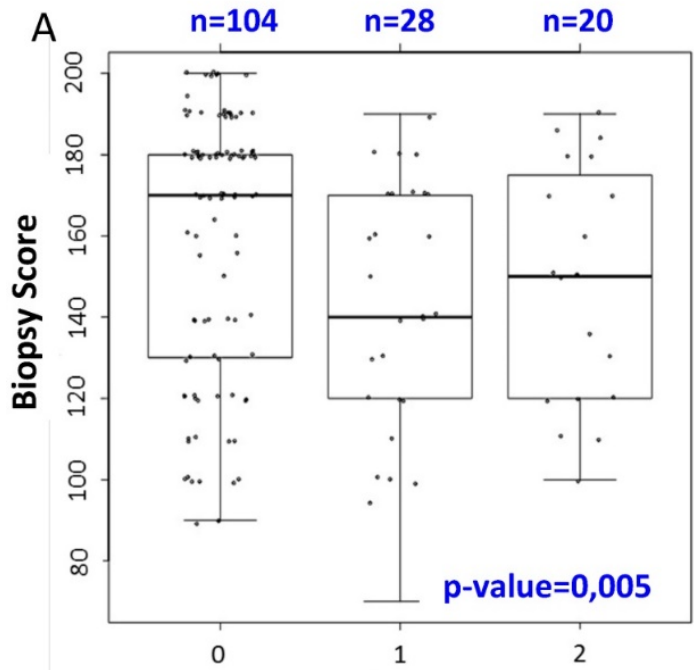

Lymph Node Status

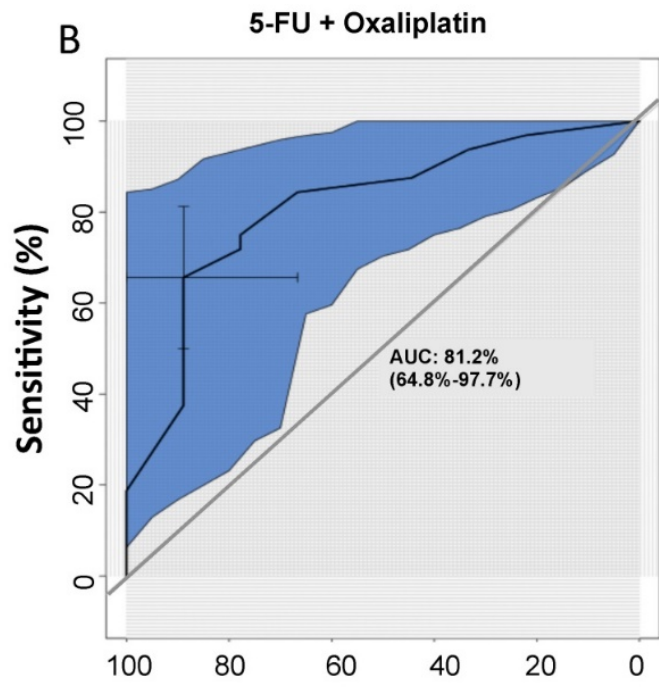

Specificity (\%)

Figure 3. A) Correlation between SRC expression in pretherapeutic biopsies and the nodal status in cytosolic staining: high SRC expression is associated with fewer lymph node metastases $(p=0.005)$. B) Correlation between pretherapeutic SRC expression under preoperative CRT with 5-FU/oxaliplatin and the nodal status: On the basis of the ROC curve (receiver operating characteristic curve) there is a high specificity and sensitivity regarding the correlation between the pretherapeutic SRC expression for patients receiving preoperative CRT with 5-FU and oxaliplatin and the nodal status. The AUC (area under curve) is $81.2 \%$, representing the quality of the test.

\section{SRC expression in resection specimens}

The expression of SRC in resection specimens correlated significantly with the development of fewer distant metastases $(p=0.048$; see figure 4$)$. Moreover we observed the trend that high SRC expression in patients who received preoperative CRT was associated with a negative nodal status $(\mathrm{p}=0.06)$. There was no statistically significant correlation between SRC expression in residual tumor tissue and DFS or local relapses during long-term follow up. In patients who received a preoperative CRT with 5-FU + oxaliplatin there was a trend for a longer CSS $(p=0.06)$ for patients with high SRC expression.

\section{Discussion}

SRC, which is a member of the SRC tyrosine kinases family, is a proto-oncogene encoding for the tyrosine kinase being localized in the cell membrane as well as in the cytosol. SRC plays a crucial role in the regulation of cell differentiation, adhesion, migration, proliferation, invasion and angiogenesis by interacting with different receptor tyrosine kinases like epidermal growth factor receptor (EGFR), human epidermal growth factor receptor 2 (HER-2), epidermal growth factor receptor 3 (HER-3) or vascular endothelial growth factor receptor (VEGFR) and being part in different cellular pathways (e.g. PI3K/Akt/Ras/Raf/MAPK). ${ }^{24,25,27,39}$ SRC inhibitors (e.g. dasatinib, bosutinib and saracatinib), which have entered the clinics, are currently being tested in phase-II-/III-trials as the tyrosine kinase represents a potential target of future oncological therapy. This analysis is the largest study so far evaluating SRC expression in pretherapeutic biopsies and corresponding resection specimens from patients with rectal cancer. In this analysis, we could not find any correlation between SRC expression and therapeutic response in terms of early surrogate parameters to preoperative CRT (TRG, tumor downsizing/ downstaging).

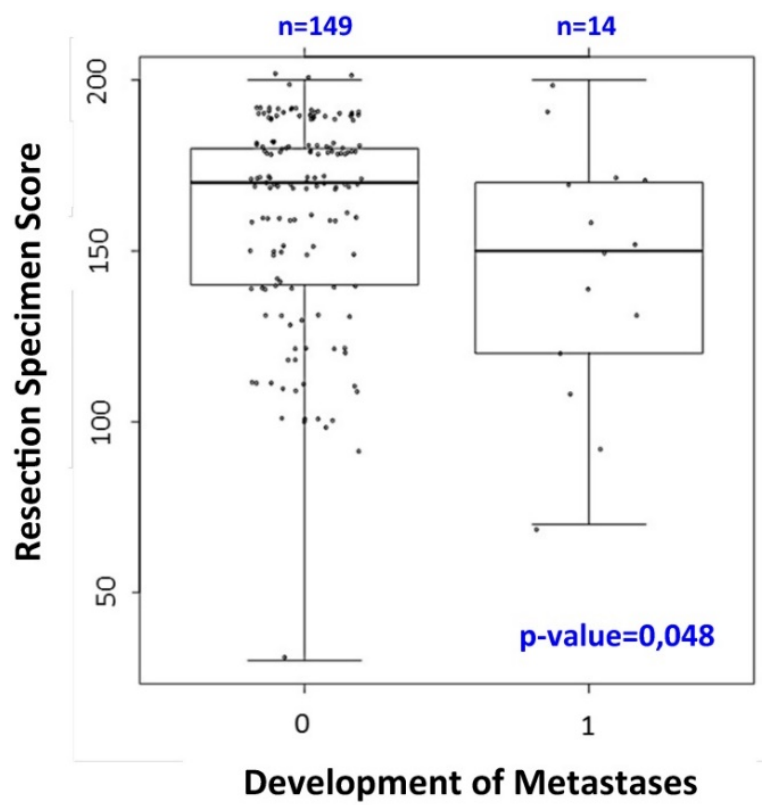

Figure 4. Correlation between cytosolic SRC expression in resection specimens and the occurrence of distant metastases: high SRC expression is associated with fewer distant metastases $(p=0.048)$. 
Regarding the prognostic potential of SRC, we could demonstrate that high SRC expression in rectal cancer was associated with a better long-term outcome. However, there is only limited data disposable considering the role of SRC in CRC.

Other studies examined the role of SRC in other cancers (e.g. breast cancer, cancer of the uterus, liver cancer). ${ }^{40-42}$ Considering these different tumor entities, high SRC expression was associated with a positive nodal status as well as with a reduced DFS and overall survival (OS). Theocharis et al. showed that SRC expression correlated with prolonged DFS and OS in patients with squamous tongue cancer. ${ }^{43}$

It is arguable whether monitoring of SRC could be a future diagnostic tool and whether SRC inhibition might improve clinical outcome under preoperative CRT, as the tyrosine kinase might represent a future therapeutic target.

Besides that, examining the role of SRC in trastuzumab resistance in HER2-positive patients could be another step towards individualized therapy strategies. Many recent studies demonstrated that SRC expression is involved in trastuzumab resistance in patients with HER-2 positive breast cancer. ${ }^{44-46}$ There is some promising data available postulating that targeting of SRC might improve the response to trastuzumab treatment in HER2-expressing cancer patients.

The TRG represents an early surrogate parameter.6,47, besides the pathological determined lymph node status ( $\mathrm{ypN}$ ) being the most important prognostic factor in rectal cancer patients. ${ }^{48}$ It is debatable whether a high SRC expression in combination with an intermediate TRG regression (TRG 2-3) and a negative nodal status in rectal cancer patients could be associated with a better prognosis and would not need any further therapeutic steps, while patients with a positive nodal status (ypN+) and a low SRC expression would postoperatively require intensified therapy. In the future the role of SRC as therapeutic target in particular in HER-2 overexpressing tumors should be further investigated based on the assumption that SRC expressing cancer cells are hyperproliferative.

An analysis by Perez et al. could demonstrate the importance of SRC and its inhibitors stating that the inhibition of SRC sensitized liver metastatic colorectal carcinoma to oxaliplatin. ${ }^{49}$ Interestingly, in our study population, patients with a high pretreatment SRC expression seemed to have benefited from a treatment schedule including oxaliplatin. Furthermore, Kopetz et al. showed that higher levels of phosphorylated SRC (pSRC) in patients with hepatic metastasized colorectal cancer were significantly associated with shorter relapse-free survival. ${ }^{50}$ In contrast to our analysis, metastatic tissue and phosphorylated SRC were analyzed. It is therefore possible that a different activation state of SRC has a different impact on clinicopathological parameters and patient survival. 51 Earlier studies also revealed a significantly higher SRC activity in tissue of hepatic metastases compared to the corresponding primary tumor in vivo which might explain different findings. ${ }^{52}$

Modifications like phosphorylation or dephosphorylation can affect the kinase activity of SRC. While we examined not specifically the significance of phosphorylated versus unphosphorylated SRC expression in tissue, further examinations with this distinction would be desirable in order to compare different SRC kinase activity states with prognostic parameters.

In order to implement pretherapeutic SRC expression level assessment into clinical practice, prospective validations of this study with larger patient cohorts would be essential. Detecting further markers for the patient's individual risk for cancer relapse and stratification of patients for the postoperative treatment would be desirable. The prospectively randomized clinical trial stratification of patients for the preoperative CRT regimen according to SRC expression would be the next step towards a risk-adapted, more individualized multimodal therapy.

In conclusion, this study evaluated the predictive and prognostic role of SRC in locally advanced rectal cancer (UICC stages II and III) treated with multimodal preoperative 5-FU-based CRT. The analyses show a favorable prognosis in patients with high SRC expression in pretherapeutic tissue as well as in resection specimens. High SRC expression in rectal cancer seems to be associated with a better long-term outcome. This finding could help in the future to stratify patients according to pretreatment SRC expression for a recurrence risk adapted preoperative treatment and should be validated and further analyzed within clinical trials. Furthermore its potential as a new therapeutic target in CRC should be evaluated. So far, SRC inhibition with e.g. saracatinib did not show a satisfying treatment success as a single agent in previously treated metastatic CRC patients ${ }^{53}$, however is currently being tested in multiple clinical trials for different entities of advanced cancer ${ }^{54,55}$ and might be a valuable addition to combination therapies for rectal cancer patients in the future.

Due to the limited data of SRC expression in rectal cancer tissue, our findings should be further validated and evaluated in large other cohorts of patients. Furthermore, our analysis is of retrospective nature and prospective studies would be desirable. 


\section{Abbreviations}

CRT: chemoradiotherapy; 5-FU: 5-fluorouracil; CSS: cancer-specific survival; CRC: colorectal cancer; UICC: Union International contre le Cancer; TME: total mesorectal excision; CTx: chemotherapy; DFS: disease-free survival; CT: computer tomography; MRI: magnetic resonance imaging; UMG: University Medical Center Göttingen; MV: megavolt; TRG: tumor regression grading; pCR: pathological Complete Response; RT: radiotherapy; EGFR: epidermal growth factor receptor; HER-2: human epidermal growth factor receptor 2; HER-3: human epidermal growth factor receptor 3; VEGFR: vascular endothelial growth factor receptor; OS: overall survival; pSRC: phosphorylated SRC.

\section{Acknowledgments}

The authors thank Birgit Jünemann for her excellent technical assistance and for providing slides for immunohistochemical staining. They appreciate the participation of all patients in this study in clinical trials and also thank the collaborative study team for randomized clinical trials (Elke Stauffer, Johanna Kreutzer and Susanne Klie) for patient monitoring and data management.

\section{Competing Interests}

The authors have declared that no competing interest exists.

\section{References}

1. Siegel RL, Miller KD, Jemal A. Cancer statistics. CA Cancer J Clin. 2016; 66: 7-30.

2. Ferlay J, Steliarova-Foucher E, Lortet-Tieulent J, et al. Cancer incidence and mortality patterns in Europe: estimates for 40 countries in 2012. Eur J Cancer. 2013; 49: 1374-403.

3. Sauer R, Becker H, Hohenberger W, et al. Preoperative versus postoperative chemoradiotherapy for rectal cancer. N Engl J Med. 2004; 351: 1731-40.

4. MacFarlane JK, Ryall RD, Heald RJ. Mesorectal excision for rectal cancer. Lancet. 1993; 341: 457-60.

5. Rödel C, Graeven U, Fietkau R, et al Oxaliplatin added to fluorouracil-based preoperative chemoradiotherapy and postoperative chemotherapy of locally advanced rectal cancer (the German CAO/ARO/AIO-04 study): final results of the multicentre, open-label, randomised, phase 3 trial. Lancet Oncol. 2015; 16: 979-89.

6. Rodel C, Martus P, Papadoupolos T, et al. Prognostic significance of tumor regression after preoperative chemoradiotherapy for rectal cancer. J Clin Oncol. 2005; 23: 8688-96.

7. Bosset JF, Collette L, Calais G, et al. Chemotherapy with preopreative radiotherapy in rectal cancer. N Engl J Med. 2006; 355: 1114-23.

8. Gerard JP, Conroy T, Bonnetain F, et al. Preoperative radiotherapy with or without concurrent fluorouracil and leucovorin in T3-4 rectal cancers: results of FFCD 9203. J Clin Oncol. 2006; 24: 4620-25.

9. Sauer R, Liersch T, Merkel S, et al. Preoperative versus postoperative chemoradiotherapy for locally advanced rectal cancer: Results from the German CAO/ARO/AIO-94 randomized phase III trial after a median follow up of 11 years. J Clin Oncol. 2012; 30: 1926-33.

10. Kopetz S, Chang GJ, Overman MJ, et al. Improved survival in metastatic colorectal cancer is associated with adoption of hepatic resection and improved chemotherapy. J Clin Oncol. 2009; 27: 3677-83.

11. Bujko K, Michalski W, Kepka L, et al. Association between pathologic response in metastatic lymph nodes after preoperative chemoradiotherapy and risk of distant metastases in rectal cancer: an analysis of outcomes in a randomized trial. Int J Radiat Oncol Biol Phys. 2007; 67: 369-77.

12. Manfredi S, Lepage C, Hatem C, Coatmeur O, Faivre J, Bouvier AM. Epidemiology and management of liver metastases from colorectal cancer. Ann Surg. 2006; 244: 254-9.
13. Gaedcke J, Liersch T, Hess C, Becker H, Rödel C, Ghadimi BM. Rectal cancer: current status of multimodal therapy- when and how? Zentralblb Chir. 2011; 136: 334-42.

14. George B, Kopetz S. Predictive and prognostic markers in colorectal cancer. Curr Oncol Rep. 2011; 13: 206-15.

15. Huh JW, Lee JH, Kim HR. Pretreatment expression of 13 molecular markers as a predictor of tumor responses after neoadjuvant chemoradiation in rectal cancer. Ann Surg. 2014; 259: 508-15.

16. Koopman M, Venderbosch S, Nagtegaal ID, van Krieken JH, Punt CJ. A review on the use of molecular markers of cytotoxic therapy for colorectal cancer, what have we learned? Eur J Cancer. 2009; 45: 1935-49.

17. Rodel C, Sauer R. Integration of novel agents into combined-modality treatment for rectal cancer patients. Strahlenther Onkol. 2007; 183: 227-35.

18. Rödel $\mathrm{C}$, Liersch $\mathrm{T}$, Becker $\mathrm{H}$, et al. Preoperative chemoradiotherapy and postoperative chemotherapy with fluorouracil and oxaliplatin versus fluorouracil alone in locally advanced rectal cancer: initial results of the German CAO/ARO/AIO-04 randomised phase 3 trial. Lancet Oncol. 2012; 13: 679-87.

19. Aschele C, Cionini L, Lonardi S, et al. Primary tumor response to preoperative chemoradiation with or without oxaliplatin in locally advanced rectal cancer: pathologic results of the STAR-01 randomized phase III trial. J Clin Oncol. 2011; 29: 2773-80.

20. Gérard JP, Azria D, Gourgou-Bourgade S, et al. Comparison of two neoadjuvant chemoradiotherapy regimens for locally advanced rectal cancer: results of the phase III trial ACCORD 12/0405-Prodige 2. J Clin Oncol. 2010; 28: $1638-44$

21. Roh MS, Yothers GA, O'Connell MJ, et al. The impact of capecitabine and oxaliplatin in the preoperative multimodality treatment in patients with carcinoma of the rectum: NSABP R-04. J Clin Oncol. 2011; 29: abstr3503.

22. Schmoll HJ, Haustermans K, Price T, et al. Preoperative chemoradiotherapy and postoperative chemotherapy with capecitabine and oxaliplatin vs. capecitabine alone in locally advanced rectal cancer: disease free survival results at interim analysis. J Clin Oncol. 2014; 32: abstr3501.

23. Martin GS. The hunting of the Src. Nature Rev Mol Cell Biol. 2001; 2: 467-75.

24. Yeatman TJ. A renaissance for SRC. Nat Rev Cancer. 2004; 4: 470-80.

25. Irby RB, Yeatman TJ. Role of Src expression and activation in human cancer. Oncogene. 2000; 19: 5636-42.

26. Summy JM, Gallick GE. Src family kinases in tumor progression and metastasis. Cancer Metastasis Rev. 2003; 22: 337-58.

27. Taylor SJ, Shalloway D. Src and the control of cell division. Bioessays. 1996; 18: 9-11.

28. Sicheri F, Kuriyan J. Structures of Src-family tyrosine kinases. Curr Opin Struct Biol. 1997; 7: 777-85.

29. Rodel C. Radiotherapy: Preoperative chemoradiotherapy for rectal cancer. Nat Rev Clin Oncol. 2010; 7: 129-30.

30. Rodel C, Liersch T, Hermann RM, et al. Multicenter phase II trial of chemoradiation with oxaliplatin for rectal cancer. J Clin Oncol. 2007; 25: $110-17$

31. Pox C, Aretz S, Bischoff SC, et al. S3-guideline colorectal cancer version 1.0. Z Gastroenterol. 2013; 51: 753-854

32. Chavaudra J. Last ICRU recommendations for the prescription, recording and reporting of external bean therapy. Cancer Radiother. 1998; 2: 607-14

33. Vorwerk H, Liersch T, Rothe H, Ghadimi M, Christiansen H, Hess CF, Hermann RM. Gold markers for tumor localization and target volume delineation in radiotherapy in rectal cancer. Strahlenther Oncol. 2009; 185: 127-33.

34. Dworak O, Keilholz L, Hoffmann A. Pathological features of rectal cancer after preoperative radiochemotherapy. Int J Colorectal Dis. 1997; 12: 19-23.

35. Brierley JD, Greene FL, Sobin LH, Wittekind C. The "y $\mathrm{y}^{\prime \prime}$ symbol: an important classification tool for neoadjuvant cancer treatment. Cancer. 2006; 106: 2526-7.

36. Sobin LH, Compton CC. TNM seventh edition: what's new, what's changed: communication from the International Union Against Cancer and the American Joint Committee on Cancer. Cancer. 2010; 116: 5336-9.

37. Berthold MR, Cebron N, Dill F, et al. KNIME: The Konstanz Information Miner. In Studies in Classification, Data Analysis, and Knowledge Organization. Springer-Verlag; 2007

38. Goulding H, Pinder S, Cannon P, et al. A new immunohistochemical antibody for the assessment of estrogen receptor status on routine formalin-fixed tissue samples. Hum Pathol. 1995; 26: 291-4.

39. Lieu C, Kopetz S. The SRC family protein tyrosine kinases: a new promising target for colorectal cancer therapy. Clin Colorectal Cancer. 2010; 9: 89-94.

40. Zhang L, Teng Y, Zhang Y, et al. c-Src expression is predictive of poor prognosis in breast cancer patients with bone metastasis, but not in patients with visceral metastasis. APMIS. 2012; 120: 549-57.

41. Hou T, Xiao J, Zhang H, Gu H, Feng Y, Li J. Phosphorylated c-Src is a novel predictor for recurrence in cervical squamous cell cancer patients. Int J Clin Exp Pathol. 2013; 6: 1121-7.

42. Chen ML, Chai CY, Yeh KT, Wang SN, Tsai CJ, Yeh YT, Yang SF. Crosstalk between activated and inactivated $\mathrm{c}$-Src in hepatocellular carcinoma. Dis Markers. 2011; 30: 325-33.

43. Theocharis S, Klijanienko J, Giaginis C, Alexandrou P, Patsouris E, Sastre-Garau X. FAK and Src expression in mobile tongue squamous cell carcinoma: associations with clinicopathological parameters and patients survival. J Cancer Res Clin Oncol. 2012; 138: 1369-77. 
44. Zhuang G, Brantley-Sieders DM, Vaught D, et al. Elevation of receptor tyrosine kinase EphA2 mediates resistance to trastuzumab therapy. Cancer Res. 2010; 70: 299-308.

45. Zhang $\mathrm{S}$, Huang $\mathrm{WC}, \mathrm{Li} \mathrm{P}$, et al. Combating trastuzumab resistance by targeting SRC, a common node downstream of multiple resistance pathways. Nature Medicine. 2011; 17: 461-9.

46. Wang SE, Xiang B, Zent R, Quaranta V, Pozzi A, Arteaga CL. Transforming growth factor beta induces clustering of HER2 and integrins by activating Src-focal adhesion kinase and receptor association to the cytoskeleton. Cancer Res. 2009; 69: 475-82.

47. Fokas E, Liersch $\mathrm{T}$, Fietkau $\mathrm{R}$, et al. Tumor regression grading after preoperative chemoradiotherapy for locally advanced rectal carcinoma revisited: updated results of the CAO/ARO/AIO-94 trial. J Clin Oncol. 2014; 32: 1554-62.

48. Chang GJ, Rodriguez-Bigas MA, Eng C, Skibber JM. Lymph node status after neoadjuvant radiotherapy for rectal cancer is a biologic predictor of outcome. Cancer. 2009; 115: 5432-40.

49. Perez M, Lucena-Cacace A, Marín-Gómez LM, et al. Dasatinib, a Src inhibitor, sensitizes liver metastatic colorectal carcinoma to oxaliplatin in tumors with high levels of phospho-Src. Oncotarget. 2016; doi: 10.18632/oncotarget.8880

50. Kopetz S, Morris VK, Parikh N, et al. Src activity is modulated by oxaliplatin and correlates with outcomes after hepatectomy for metastatic colorectal cancer. BMC Cancer. 2014; doi: 10.1186/1471-2407-14-660

51. Gargalionis AN, Karamouzis MV, Papavassiliou AG. The molecular rationale of Src inhibition in colorectal carcinomas. Int J Cancer. 2014 May 1;134(9):2019-29. doi: 10.1002/ijc.28299.

52. Talamonti MS, Roh MS, Curley SA, Gallick GE. Increase in activity and level of pp60c-src in progressive stages of human colorectal cancer. J Clin Invest. 1993; 91:53-60.

53. Reddy SM, Kopetz S, Morris J et al. Phase II study of saracatinib (AZD0530) in patients with previously treated metastatic colorectal cancer. Invest New Drugs. 2015; 33: 977-84.

54. Chee CE, Krishnamurthi S, Nock CJ et al. Phase II study of dasatinib (BMS-354825) in patients with metastatic adenocarcinoma of the pancreas. Oncologist. 2013; 18: 1091-92.

55. Strickler JH, McCall S, Nixon AB et al. Phase I study of dasatinib in combination with capecitabine, oxaliplatin and bevacizumab followed by an expanded cohort in previously untreated metastatic colorectal cancer. Invest New Drugs. 2014; 32: 330-39. 